\title{
PERFIL DE SENSIBILIZAÇÃO A ALÉRGENOS DOMICILIARES EM PACIENTES AMBULATORIAIS
}

\author{
Fernanda Aparecida Almeida Soares, Gesmar Rodrigues Silva Segundo, Ronaldo Alves, Leandro Hideki Ynoue, Rafael Oliveira Resende, Mônica \\ Camargo Sopelete, Deise Aparecida Oliveira Silva, Sun-sang Joseph Sung, Ernesto A. Taketomi* \\ Trabalho realizado na Unidade de Pesquisa de Alergia e Imunologia Clínica do Instituto de Ciências Biomédicas da Universidade Federal de \\ Uberlândia, MG.
}

\author{
*Correspondência: \\ Unidade de Pesquisa em \\ Alergia e Imunologia Clínica \\ Universidade Federal de \\ Uberlândia \\ Avenida Pará, 1720. Bloco \\ C, Campus Umuarama \\ Uberlândia/MG \\ Cep: $38400-902$ \\ Tel: (34) 3218-2195 \\ Fax: (34) 3218-2333 \\ taketomi@ufu.br
}

\begin{abstract}
RESUMO
Oвjetrvo. Conhecer o perfil de sensibilização dos pacientes com diagnóstico de doenças alérgicas atendidos no Ambulatório de Alergia do Hospital de Clínicas da Universidade Federal de Uberlândia.

Métodos. Foi realizado um estudo retrospectivo por meio da análise de prontuários de pacientes atendidos no serviço, que foram submetidos ao teste cutâneo de puntura (TCP) para os alérgenos de Dermatophagoides pteronyssinus (Der $\mathrm{p}$ ), Dermatophagoides farinae (Der f), Blomia tropicalis (Blo t), Canis familiaris (Can f), Felis domesticus (Fel d), Blattella germanica (Bla g) e Alternaria alternata, e respondido corretamente ao questionário ISAAC.

Resultados. Foram analisados 212 prontuários de pacientes que preenchiam os critérios de inclusão. A rinite alérgica isoladamente foi a maior causa de atendimento (32\%), seguida das associações asma e rinite $(29,7 \%)$ e asma, rinite e eczema (9,4\%). Pacientes com asma isoladamente perfizeram apenas 1,9\% dos atendimentos. A sensibilização dos pacientes observada foi de 73,5\%, sendo de $61,7 \%$ para Der p, 59,9\% para Der f, 54,7\% para Blo t, 45,7\% para Bla g, 38,2\% para Can f, 33,3\% para Fel de $9,9 \%$ para Alternaria. Não houve diferenças significantes entre as patologias encontradas e os perfis de sensibilização.
\end{abstract}

Conclusão. Os principais alérgenos sensibilizantes determinados pelo TCP foram os ácaros, com predomínio de Der p e Der f, chamando a atenção a elevada prevalência de sensibilização ao último. Ainda foi observada elevada sensibilização aos alérgenos de B. germanica, superior aos estudos anteriores realizados no Pás.

Unitermos: Sensibilização alergênica. Alérgenos domiciliares. Asma. Rinite alérgica. Dermatite atópica. Teste cutâneo.

\section{INTRODUÇÃo}

As doenças alérgicas apresentaram um expressivo aumento na sua prevalência e na morbidade nas últimas décadas'. Esse aumento do número de doentes e da sensibilização a alérgenos na asma e rinite alérgicas em indivíduos atópicos poderia ser explicado por diversos fatores, entre os quais, melhor reconhecimento da doença; maior reatividade imunológica; maior exposição ambiental; mudanças no estilo de vida, com maior permanência em ambientes fechados e modificações dos hábitos alimentares; contribuição de fatores infecciosos e socioeconômicos e concentração exacerbada de poluentes externos ${ }^{2-4}$. Segundo dados do International Study of Asthma and Allergy in Childhood (ISAAC) no Brasil, a prevalência de asma pode atingir até $28,2 \%$ da população, dependendo do centro estudado ${ }^{5}$.

A ampliação dos conhecimentos relacionados às doenças alérgicas mostra uma distinção de cenários de acordo com regiões analisadas. 0 perfil de sensibilização dos pacientes sofre influências locais, das questões relacionadas às condições climáticas e flora local. A análise de alérgenos em diferentes locais no Brasil mostra uma diferença entre os achados, variando com as condições de temperatura e umidade do ar, tanto do microclima de ambientes fechados como das condições externas ${ }^{6-7}$. Estudos realizados na região central do país mostraram alta prevalência de Dermatophagoides farinae $e^{8-9}$, enquanto em algumas regiões litorâneas existe uma preponderância de Dermatophagoides pteronyssinus ${ }^{10-12}$. Estudos em automóveis demonstraram grandes quantidades de alérgenos de animais, tanto em transportes públicos como em carros particulares, ${ }^{6,13}$.

A sensibilização alérgica dos indivíduos depende da interação entre os fatores genéticos e ambientais, e reforça a importância da exposição alergênica para o desenvolvimento das doenças alérgicas ${ }^{14,15}$. Entre $70 \%$ e $85 \%$ dos pacientes com diagnóstico de asma e rinite alérgica possuem algum tipo de sensibilização a aeroalérgenos. A realização dos testes cutâneos ou dosagem sérica de lgE espećfica é parte importante do diagnóstico, bem como do tratamento.

O objetivo deste estudo foi conhecer o perfil de sensibilização dos pacientes com diagnóstico de doenças alérgicas atendidos no Ambulatório de Alergia e Imunologia do Hospital de Clínicas da Universidade Federal de Uberlândia (HC-UFU)

\section{Métodos}

Foi realizado um estudo retrospectivo em que foram analisados os prontuários contendo o questionário ISAAC ${ }^{16}$ aplicados aos pacientes atendidos no Ambulatório de Alergia e Imunologia do HC-UFU e submetidos a testes cutâneos de puntura (TCP) de leitura imediata para avaliação da sensibilidade a aeroalérgenos, no período de 20 de maio de 2002 a 24 de janeiro de 2005. Para a realização do TCP, foram excluídos pacientes com dermografismo e em uso de medicações 
anti-histamínicas, corticosteróides orais ou antileucotrienos nas três semanas antecedentes à realização do teste. Foram selecionados apenas pacientes cujos formulários continham informações que preenchiam todos os critérios de inclusão.

O TCP foi realizado conforme descrição de Godinho et al. ${ }^{17}$, com aplicação de uma gota do alérgeno no antebraço do paciente, seguida de perfuração superficial da pele com puntor apropriado. Foram utilizados os extratos alergênicos de Dermatophagoides pteronyssinus (Der p), Dermatophagoides farinae (Der f), Blomia tropicalis (Blo t), Blattella germanica (Bla g), Alternaria alternata e epitélios de Canis familiaris (Can f) e Felis domesticus (Fel d). Todos os extratos alergênicos foram procedentes da Bayer Corporation, Spokane, Washington, EUA, e utilizados nas concentrações recomendadas pelos fornecedores. Como controle positivo, foi utilizado cloridrato de histamina (I mg/ml; Bayer Corporation) em solução salina fisiológica com glicerol a 50\%, sendo que este último foi usado como controle negativo da reação. O critério de positividade adotado foi a presença de pápula igual ou superior a $3 \mathrm{~mm}$, obtida pela média de dois diâmetros perpendiculares, ocorrida em 15 a 20 minutos após a aplicação do alérgeno. $O$ grau de positividade era dado pela comparação do tamanho, elevação e hiperemia com o controle positivo. O teste foi realizado por técnicos de nível superior do Ambulatório de Alergia e Imunologia do HC-UFU.

Para todos os cálculos estatísticos foi utilizado o software GraphPad Prism versão 4.0. Todos os resultados foram considerados estatisticamente significativos a um nível de significância de $5 \%(p<0,05)$.

\section{Resultados}

Foram analisados 257 prontuários que continham o questionário ISAAC de pacientes atendidos no Ambulatório de Alergia e Imunologia do HC-UFU, sendo que em 212 o questionário e o TCP estavam devidamente preenchidos.

Dos 2 I 2 prontuários, I4I (66,5\%) pacientes pertenciam ao sexo feminino e 7 I (33,5\%) eram do sexo masculino, com idade mediana de 24 anos, variando de 4 a 65 anos. As patologias encontradas em ordem decrescente de freqüência foram a rinite alérgica $(79,7 \%)$, asma (43,3\%) e dermatite atópica (24,5\%) (Tabela I).

A rinite isoladamente foi a maior causa de atendimento (32\%), seguida da associação asma e rinite, responsável por 29,7\% dos atendimentos, e, posteriormente, associação asma, rinite e eczema, com $9,4 \%$ dos atendimentos. Pacientes com asma isoladamente perfizeram apenas 1,9\% dos atendimentos, enquanto aqueles apenas com dermatite atópica foram $4,7 \%$ do total.

O TCP foi positivo em I $56(73,5 \%)$ e negativo em $56(26,4 \%)$ aos pacientes para a bateria de extratos analisada. A freqüência de positividade aos aeroalérgenos analisados foi: 13 I $(61,7 \%)$ para Der $p$, I 27 (59,9\%) para Der f, I I 6 (54,7\%) para Blo t, 97 (45,7\%) para Bla g, 81 (38,2\%) para Can f, 70 (33,3\%) para Fel d e 21 (9,9\%) para Alternaria, conforme Figura I. Houve diferença significativa pelo teste do Qui-quadrado analisando o conjunto de alérgenos como um todo $(p<0,000 \mathrm{I})$. Não houve diferença significativa entre os alérgenos das diferentes espécies de ácaros, nem em relação aos ácaros e barata. Os alérgenos de animais domésticos tiveram positividade ao TCP estatisticamente menor que os de ácaros, e somente Fel d foi significativamente menor que Bla g. A positividade ao alérgeno de Alternaria foi menor que todos os outros analisados.

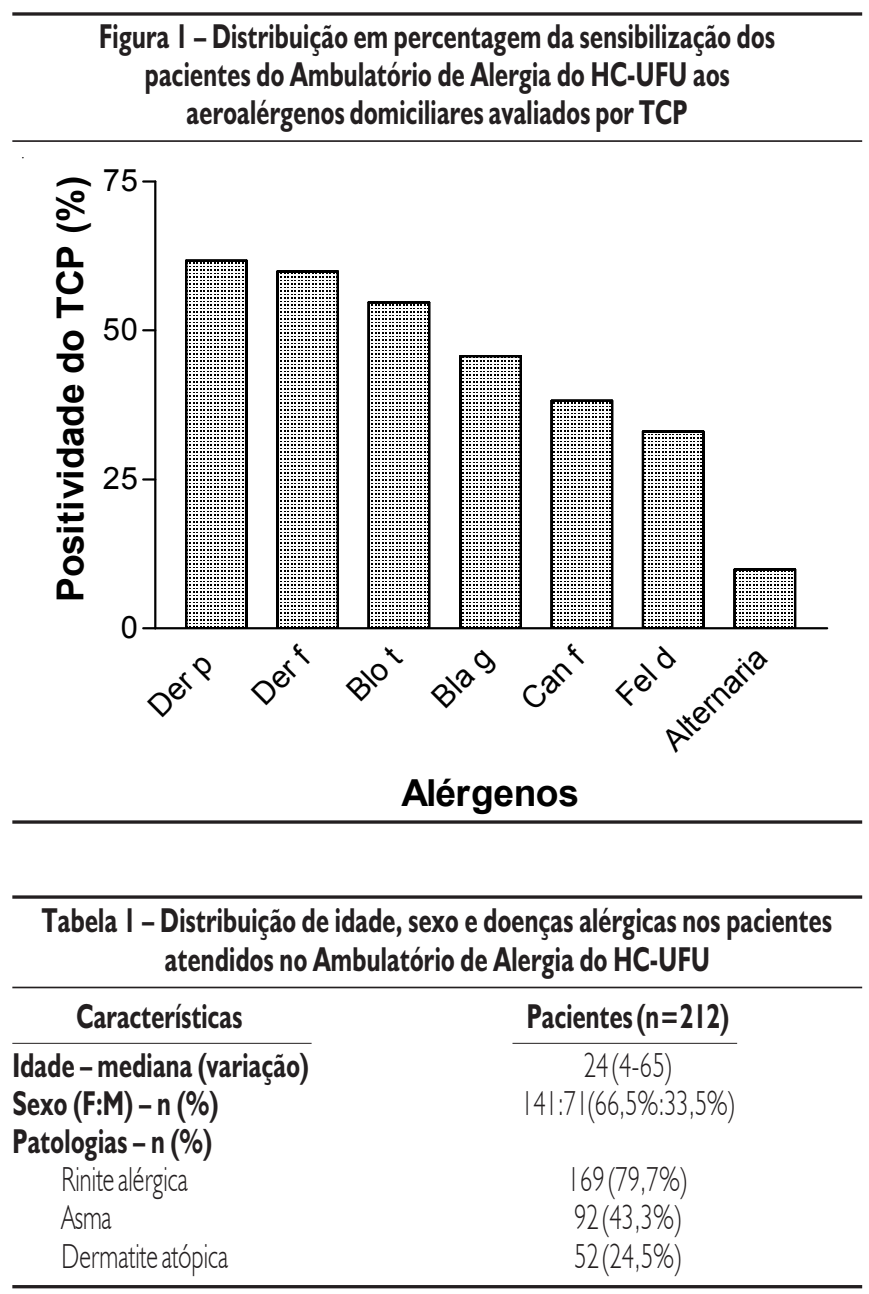

A maioria dos pacientes com teste positivo estava sensibilizada a mais de um alérgeno, sendo comuns múltiplas sensibilizações (Tabela 2). A distribuição de positividade do TCP de acordo com as patologias está descrita na Tabela 3. Não houve diferença significativa entre a positividade do TCP nas patologias analisadas $(p=0,15 \mid 2)$. Os testes cutâneos positivos foram estratificados de acordo com cada antígeno testado, e sua prevalência calculada em relação ao número de indivíduos pertencentes a cada patologia separadamente ou associada. Analisando as patologias isoladas, houve diferença significativa entre asma, rinite e dermatite atópica. Pacientes que apresentaram positividade ao TCP com diagnóstico apenas de dermatite atópica foram significativamente menores que aqueles com diagnóstico isolado de asma $(p=0,0253)$ e de rinite $(p=0,0730)$.

\section{Discussão}

A sensibilização a aeroalérgenos depende da inter-relação entre a susceptibilidade genética e a exposição a alérgenos. As condições climáticas e os aspectos culturais locais podem predispor a sensibilização por diferentes aeroalérgenos, de acordo com a região estudada. Sendo assim, o conhecimento desses perfis regionais é importante para traçar medidas de controle ambiental e indicação de imunoterapia. 


\begin{tabular}{ccc}
\hline Tabela 2 - Distribuição de pacientes por número de alérgenos sensibilizantes \\
\hline Número de & \multicolumn{2}{c}{ Pacientes } \\
\cline { 2 - 3 } alérgenos & (n) & $(\%)$ \\
sensibilizantes & 10 & 4,7 \\
1 & 21 & 9,9 \\
2 & 30 & 14,1 \\
3 & 23 & 10,8 \\
4 & 36 & 16,9 \\
5 & 25 & 11,7 \\
6 & 11 & 5,1 \\
7 & 56 & 26,4 \\
nenhum &
\end{tabular}

\begin{tabular}{|c|c|c|}
\hline \multirow[t]{2}{*}{ Patologia } & Teste positivo & Teste negativo \\
\hline & $\begin{array}{l}\mathrm{N}(\%) \\
\end{array}$ & $N(\%)$ \\
\hline $\operatorname{Rinite}(n=169)$ & $125(73,9)$ & $44(26,1)$ \\
\hline $\operatorname{Asma}(n=92)$ & $72(78,2)$ & $20(21,8)$ \\
\hline Dermatite $(n=52)$ & $33(63,4)$ & $19(36,6)$ \\
\hline
\end{tabular}

Estudos anteriores sobre população com essas mesmas patologias apresentaram percentagem de sensibilização em valores aproximados aos achados. Assim, estudos realizados na cidade do Rio de Janeiro, RJ, mostraram uma positividade de $67,5 \%$ ao TCP em pacientes com rinite, com ou sem asma ${ }^{18}$ e outro, em pacientes com sintomas sugestivos de rinite, verificou uma positividade a poeira domiciliar de 74,9\% ${ }^{17}$. Na cidade de Belo Horizonte, MG, foram avaliadas 227 crianças e adolescentes com história de asma ou rinite, demonstrando positividade dos testes cutâneos em $90 \%$ dos pacientes $^{19}$. Rosário mostrou uma prevalência de $80 \%$ de positividade ao TCP em pacientes com asma e/ou rinite ${ }^{20}$. Um estudo multicêntrico brasileiro que avaliou a sensibilização a alérgenos inalantes e alimentares em crianças atópicas utilizando dosagem de lgE específica mostrou sensibilização em 79\%, considerando como ponto de corte níveis iguais ou maiores que classe ${ }^{21}$. Um estudo prévio do nosso grupo, que analisou comparativamente o TCP, testes intradérmicos e a dosagem de lgE específica por ELISA (enzyme-linked immunosorbent assay) em 89 indivíduos asmáticos, também na cidade de Uberlândia, MG, demonstrou uma sensibilização de $73 \%$ ao TCP22. Os resultados encontrados no presente estudo mostram uma sensibilização a aeroalérgenos $(73,5 \%)$ similar aos demais estudos apresentados.

Quando comparamos os dados de múltipla sensibilização, apenas Naspitz et al. ${ }^{21}$ descreveram dados semelhantes, demonstrando que a sensibilização a mais de um alérgeno foi observada em $85 \%$ dos pacientes com IgE sérica positiva; valor elevado, porém, inferior ao observado nesta pesquisa, que foi de 93,6\% (I 46/I56 pacientes).

Em estudos prévios realizados sobre perfis de sensibilização no Brasil, D. pteronyssinus é o ácaro com maior associação à positividade do teste, exceto nas descrições de Sarinho et al. ${ }^{23}$ e de Rosário ${ }^{20}$, em que houve um predomínio de $B$. tropicalis. Alguns estudos prévios não avaliaram a sensibilização ao extrato de
D. farinae, e verificamos diferenças de acordo com a região de estudo. A porcentagem de indivíduos sensibilizados a $D$. farinae em nosso estudo foi similar a $D$. pteronyssinus, dado semelhante ao descrito anteriormente por outros autores ${ }^{19,21}$, porém, diferente do descrito por outros $10,11,20,24$. Esta diferença pode ser atribuída a alterações climáticas, uma vez que o ácaro $D$. farinae tem melhor desenvolvimento em regiões de maior temperatura e menor umidade, como em nossa cidade. A maior sensibilização a alérgenos de $D$. farinae coincide com a maior exposição a esse alérgeno demonstrada previamente $8,9,25$.

A sensibilização aos alérgenos de Blattella germanica em nosso estudo chegou a 45,7\%, acima da demonstrada por outros autores, que variou entre $15 \%$ e $34,4 \% 18,21$. Questionamos se a maior sensibilização demonstrada no presente estudo aos alérgenos de barata possa estar relacionada à reatividade cruzada com ácaros ou se é decorrente de uma maior exposição da população estudada a esses alérgenos. Além disso, a reatividade cruzada pode ser resultante da sensibilização a estruturas altamente conservadas entre os invertebrados, tais como tropomiosina de baratas, camarões, lagostas, caranguejos, lulas, molus $\cos ^{26,27}$ ou paramiosina de Schistosoma mansoni e Onchocerca volvulus ${ }^{28}$. A fim de elucidar a elevada positividade do TCP ao alérgeno de barata, devemos ampliar este estudo, para verificação da existência de reatividade cruzada.

Os alérgenos de animais são outros importantes desencadeadores da doença alérgica; neste estudo, entretanto, apresentaram positividade inferior aos alérgenos de barata. Outros autores encontraram sensibilização variando entre $8,8 \%$ e $32,7 \%$ para alérgenos de cão e de $12,2 \%$ a $16,9 \%$ para alérgenos de gato, similares aos dados obtidos neste estudo 17,21 .

A utilização de extratos padronizados é importante na avaliação da sensibilização dos pacientes, uma vez que os dados obtidos serão confiáveis e de maior reprodutibilidade, além de serem a base para a realização da imunoterapia específical' ${ }^{14}$

\section{Conclusão}

Os achados obtidos neste perfil de reatividade cutânea corroboram a importância da sensibilização a aeroalérgenos na patogênese das doenças alérgicas. A necessidade da implementação de uma estratégia global de medidas de controles ambientais em conjunto com o tratamento medicamentoso é reforçada com os dados atuais, uma vez que medidas de controle ambiental realizadas de forma isolada têm se mostrado ineficazes em alguns estudos anteriores ${ }^{29,30,31}$. Além disso, o conhecimento do perfil de sensibilização pode facilitar a realização da imunoterapia específica com alérgenos em determinados casos, particularmente naqueles em que o controle ambiental global é de difícil realização.

\section{Agradecimentos}

Trabalho financiado pelas seguintes agências de fomento brasileiras: Capes (Coordenação de Aperfeiçoamento de Pessoal de Nível Superior, Braślila, DF), CNPq (Conselho Nacional de Pesquisa e Desenvolvimento, Brasília, DF) e Fapemig (Fundação de Amparo à Pesquisa do Estado de Minas Gerais, Belo Horizonte, MG). 


\section{Conflito de interesse: não há.}

\section{SUMMARY}

\section{INDOOR ALLERGEN SENSITIZATION PROFILE IN ALLERGIC PATIENTS OF THE ALLERG CLINIC IN THE UNIVERSITY HOSPITAL IN UBERLÂNDIA, BrAZIL}

OBJECTIVE. To evaluate allergens among patients with allergic respiratory disease attended at the Allergy Clinic of the University Hospital Federal University of Uberlândia.

METHODS. A retrospective study was performed using medical records. Patients were included iftheir ISAAC questionnaires were correctly filled out and their skin prick tests were positive to at least one of the allergens from Dermatophagoides pteronyssinus(Derp), Dermatophagoidesfarinae (Derf), Blomia tropicalis (Blo t), Canis familiaris (Can f), Felis domesticus (Fel d), Blattella germanica (Bla g) and Alternaria alternata.

RESULTS. Two-hundred and twelve medical records fulfilled the inclusion criteria. Allergic rhinitis was the main clinical diagnosis (32\%), followed by concomitant manifestation of asthma and rhinitis (29.7\%), and asthma, rhinitis and atopic dermatitis (9.4\%). Asthma alone was found only in $1.9 \%$ of patients. The total sensitization observed was $73.5 \%$, of which $61.7 \%, 59.9 \%, 54.7 \%, 45.7 \%, 38.2 \%, 33.3 \%$, 9.9\% were sensitized to Der p, Der f, Blo t, Bla g, Can f, Fel d and Alternaria, respectively. No significant difference was found between allergic disease types and source of allergen sensitization.

CONCLUSION. The highest sensitization in allergic patients under study was to dust mites, especially Der $p$ and Derf. It is noteworthy that the number of patients sensitized to cockroach extract was uncommonly high when compared to previous studies. [Rev Assoc Med Bras 2007; 53(I): 25-8]

KEY wORDS: Allergen sensitization. Indoor allergen. Asthma. Allergic rhinitis. Atopic dermatitis. Skin prick test.

\section{REFERÊNCIAS}

I. Mosges R. The increasing prevalence of allergy: a challenge for the physician. Clin Exp Allergy Rev. 2002;2: I3-7.

2. Platts-Mills TA, Erwin E, Heymann P, Woodfolk I. Is the hygiene hypothesis still a viable explanation for the increased prevalence of asthma? Allergy. 2005;60(Suppl 79):25-31.

3. Ramsey CD, Celedon JC. The hygiene hypothesis and asthma. Curr Opin Pulm Med. 2005; I I: I 4-20.

4. Boutin-Forzano S, Hammou Y, Gouitaa M, Charpin D. Air pollution and atopy. Allerg Immunol. (Paris) 2005;37: I I-6.

5. Solé D, Camelo-Nunes IC, Waldensen GF, Naspitz CK, Vanna AT, Amorim A, et al. A asma em crianças é um problema de saúde pública? Rev Bras Alergia Imunopatol. 2004;27: I 85-8.

6. Justino CM, Segundo GR, Pereira FL, Silva DA, Sopelete MC, Sung SS, et al. Mite and pet allergen exposure in Brazilian private cars. Ann Allergy Asthma Immunol. 2005;94:658-61.

7. Graudenz GS, Kalil J, Latorre MR, Arruda LK, Gambale W, MoratoCastro FF. Exposição alergênica e sintomas respiratórios em ambientes climatizados. Rev Bras Alerg Imunopatol 2004; 27:94- 102.

8. Terra SA, Silva DA, Sopelete MC, Mendes J, Sung SJ, Taketomi EA. Mite allergen levels and acarologic analysis in house dust samples in Uberaba, Brazil. J Investig Allergol Clin Immunol. 2004; I 4:232-7.

9. Sopelete MC, Silva DA, Arruda LK, Chapman MD, Taketomi EA. Dermatophagoides farinae (Der $f \quad l$ ) and Dermatophagoides pteronyssinus (Der p I) allergen exposure among subjects living in Uberlandia, Brazil. Int Arch Allergy Immunol. 2000; I 22:257-63.

10. Arruda LK, Rizzo MC, Chapman MD, Fernandez-Caldas E, Baggio D, Platts-Mills TA, Naspitz CK. Exposure and sensitization to dust mite allergens among asthmatic children in Sao Paulo, Brazil. Clin Exp Allergy. 1991;21:433-9.

I I. Rullo VE, Rizzo MC, Arruda LK, Sole D, Naspitz CK. Daycare centers and schools as sources of exposure to mites, cockroach, and endotoxin in the city of Sao Paulo, Brazil. J Allergy Clin Immunol. 2002; I 1 0:582-8.

12. Dutra BM, Rosário Filho NA, Zavadniak AF. Alérgenos inaláveis em Curitiba: uma revisão de sua relevância clínica. Rev Bras Alergia Imunopatol. 2001,24: I89-195.

13. Pereira FL, Silva DA, Sopelete MC, Sung SS, Taketomi EA. Mite and cat allergen exposure in Brazilian public transport vehicles. Ann Allergy Asthma Immunol. 2004;93: 1 79-84.

14. Platts-Mills TA, Chapman MD. Dust mites: immunology, allergic disease, and environmental control. J Allergy Clin Immunol. 1987;80:755-75.

15. Simpson A, Custovic A. The role of allergen avoidance in the secondary prevention of atopic disorders.Curr Opin Allergy Clin Immunol. 2005;5:223-7.

16. Asher MI, Keil U, Anderson HR, Beasley R, Crane J, Martinez F, et al. International Study of Asthma and Allergies in Childhood (ISAAC): rationale and methods. Eur Respir J. 1995;8:483-91.

17. Godinho R, Lanza M, Godinho A, Rodrigues A, Assiz TM. Freqüência de positividade em teste cutâneo para aeroalérgenos. Rev Bras Otorrinolarigol. 2003:69:824-8.

18. Feld L, Lima BC, Costa E. Sensibilização a alérgenos inaláveis em pacientes com alergia respiratória na cidade no Rio de Janeiro: comparação entre asma brônquica e rinite isolada. Rev Bras Alergia Imunopatol. 200 I; 24:54-64.

19. Marques MC, Pinto JA, Greco DB. Sensibilização a aeroalérgenos em crianças e adolescentes atópicos em Belo Horizonte, MG: comparação e estimativa de IgE específica in vivo versus in vitro. Rev Bras Alergia Imunopatol. 2001:24:22-32.

20. Rosario NA. Sensibilización al ácaro Blomia tropicalis en pacientes con alergia respiratoria. Rev Alergia. 1992;5:96- 100.

21. Naspitz CK, Sole D, Aguiar MC, Chavarria ML, Rosario Filho N, Zuliani A, et al. Grupo PROAL. Phadiatop in the diagnosis of respiratory allergy in children: Allergy Project_PROAL. J Pediatr. (Rio J). 2004; 80:217-22.

22. Moreira MR, Mendonça MR, Gomes-Junior EA, Souza GG, Gervásio AM. Sopelete MC, et al. Sensibilização a alérgenos inaláveis domiciliares em pacientes asmáticos em Uberlândia, MG. Rev Bras Alergia Imunopatol. 2001;24:1|-21.

23. Sarinho E, Rizzo MC, Just E, Fernandez-Caldas E, Sole D. Sensibilização aos ácaros domésticos em crianças atópicas e não-atópicas de Recife, PE, Brasil. Rev Bras Alergia Imunopatol. 2000;23: 105- 13.

24. Geller M, Esch RE, Fernandez-Caldas E. Sensibilização acarina na atopia do Rio de Janeiro: considerações preliminares. An Acad Nac Med. 1993; 153: 174-5.

25. Silva MC, Justino CM, Pereira FL, Segundo GR, Silva DA, Sung-Sang JS, et al Exposição alergênica em cinemas na cidade de Goiânia, GO. Rev Bras Alergia Imunopatol. 2005; 28: 194-7.

26. Santos AB, Chapman MD, Aalberse RC, Vailes LD, Ferriani VP, Oliver C, et al. Cockroach allergens and asthma in Brazil: identification of tropomyosin as a major allergen with potential cross-reactivity with mite and shrimp allergens. J Allergy Clin Immunol. 1999; 104:329-37.

27. Reese G, Ayuso R, Lehrer SB. Tropomyosin: an invertebrate pan-allergen. Int Arch Allergy Immunol. 1999; I 19:247-58.

28. Steel C, Limberger RJ, McReynolds LA, Ottesen EA, Nutman TB. B cell responses to paramyosin. Isotypic analysis and epitope mapping of filarial paramyosin in patients with onchocerciasis. J Immunol. 1990; | 45:3917-23.

29. Woodcock A, Forster L, Matthews E, Martin J, Letley L, Vickers M, et al. Control of exposure to mite allergen and allergen-impermeable bed covers for adults with asthma. N Engl J Med. 2003;349:225-36.

30. Terreehorst I, Hak E, Oosting AJ, Tempels-Pavlica Z, De Monchy, JGR, Bruijnzeel-Koomen CAFM, et al. Evaluation of impermeable covers for bedding in patients with allergic rhinitis. N Engl J Med. 2003:349:237-46.

31. Platts-Mills TA. Allergen avoidance in the treatment of asthma and rhinitis. N Engl J Med. 2003;349:207-8.

Artigo recebido: 15/09/05

Aceito para publicação: 26/12/05 